\title{
Real-Time Fatigue Analysis of Driver through Iris Recognition
}

\author{
Gopalakrishna $\mathbf{K}^{\mathbf{1}}$, Hariprasad S. A. ${ }^{2}$ \\ Departement of Electronics and Communication Engineering, Jain University, India
}

\begin{tabular}{l} 
Article Info \\
\hline Article history: \\
Received May 13, 2017 \\
Revised Aug 21, 2017 \\
Accepted Sep 5, 2017 \\
\hline
\end{tabular}

\section{Keyword:}

Iris recognition

Eye blinking

Electrocardiogram

Electroencephalograms

\begin{abstract}
In recent days, the driver's fault accounted for about $77.5 \%$ of the total road accidents that are happening every day. There are several methods for the driver's fatigue detection. These are based on the movement of the eye ball using eye blinking sensor, heart beat measurement using Electro Cardio Gram, mental status analysis using ElectroEncephaloGram, muscle cramping detection, etc. However the above said methods are more complicated and create inconvenience for the driver to drive the vehicle. Also, these methods are less accurate. In this work, an accurate method is adopted to detect the driver's fatigue based on status of the eyes using Iris recognition and the results shows that the proposed method is more accurate (about 80\%) compared to the existing methods such as Eye blink Sensor method.
\end{abstract}

Copyright $@ 2017$ Institute of Advanced Engineering and Science. All rights reserved.

Corresponding Author:

Gopalakrishna K.,

Departement of Electronics and Communication Engineering,

School of Engineering and Technology, Jain University,

Jakkasandra (Post), Kanakapura Road, Ramanagara (District), Karnataka.

Email: k.gopalakrishna@jainuniversity.ac.in

\section{INTRODUCTION}

According to ministry of Road Transport and Highways, Government of India, Transport Research Wing Report-2015, Road accidents are recognized as serious global health problem. Nearly 5, 00,000 road accidents caused nearly 1, 46,000 deaths and left more than thrice that number injured in India in 2015 [3]. National Crime Records Bureau, Ministry of Road Transport \& Highway, Law commission of India, 1214 road crashes occur every day in India, 377 people die every day. Driver's fault accounted for a whopping $77.5 \%$ of the total road accidents while pedestrian and cyclist's fault accounted for a mere $3.7 \%$ and Defect in road condition just $1.5 \%$ and remaining is due to technical issues in the vehicles. According to Road Traffic Injuries (RTI) data, in India, half million accidents happen in a year and as per the RTI survey reports, the road accidents ranked fourth among the causes of death around the world. Around 1.3 million people die every year on the roads throughout the world and around 40 million people suffer non-fatal injuries and many of them become physically disabled. Due to this, to monitor the driver's vigilance level and to avoid fatigue driving, it is necessary for the intelligent transport system (ITS).

These high rates of accidents are due to the driver's drowsiness which leads to take correct timely actions. The different methods used to find the driver's fatigue are; Mr.Narender Kuamr and Dr. N.C. Barwar [4] describes that, Yawning is detected by monitoring the changes in the geometrical features of the mouth. This method uses eye Blinking Sensor to monitor the eye status and the limitation of this method is that, there are several chances of false alarming and hence this is not an accurate method.Mr.Anwesha Sengupta et al [5], in their paper says that, the Analysis of fatigue due to sleep deprivation using EEG synchronization is a promising field of research and they have analysed the level of fatigue in drivers due to sleep-deprivation by studying the synchronization between EEG data recorded from various brain areas by employing the state-space analysis.This method gives better results but it is complicated, if used practically, while driving.WANG He [6], describes in his paper that, the heart rate 
variability detection provides the details about the tiredness of the driver. This method is quite complicated as it needs an electrode to be connected to the heart of the driver. Hence, it makes the driver uncomfortable to drive the vehicle. M. Mischi, C. Rabotti, and M.Cardinale [7] describes about the, Electromyography (EMG) analysis and vibration analysis related to fatigue. This is method is also quite complicated and creates problem for driving.

The major limitations in the face detection are the uncontrolled illumination and the variations in the pose. To address this, Mr.Venkatarama Phani Kumar [1] describes the Modular two-dimensional Principle Component Analysis in which, the image is partitioned into four four equal segments and then the Histogram equalization is applied to reduce the illumination impact due to the varing conditions. However the accuracy of Ires recognition in presence of the noise can be improved as described by Mr.Vineet Kumar et al [2], in their paper describe about the Iris localization using edge map genetration and adaptive circular Houghtransform for less constrained Iris images.

\section{PROPOSED METHOD}

\subsection{Block Diagram}

Figure 1 shows the block diagram of the proposed system. It uses ARM 7 based LPC2148 processor, for which a camera is interfaced through the Laptop or a PC. LCD is interfaced to the ARM processor to display the status and a Buzzer is used to give the alert signal to the driver.

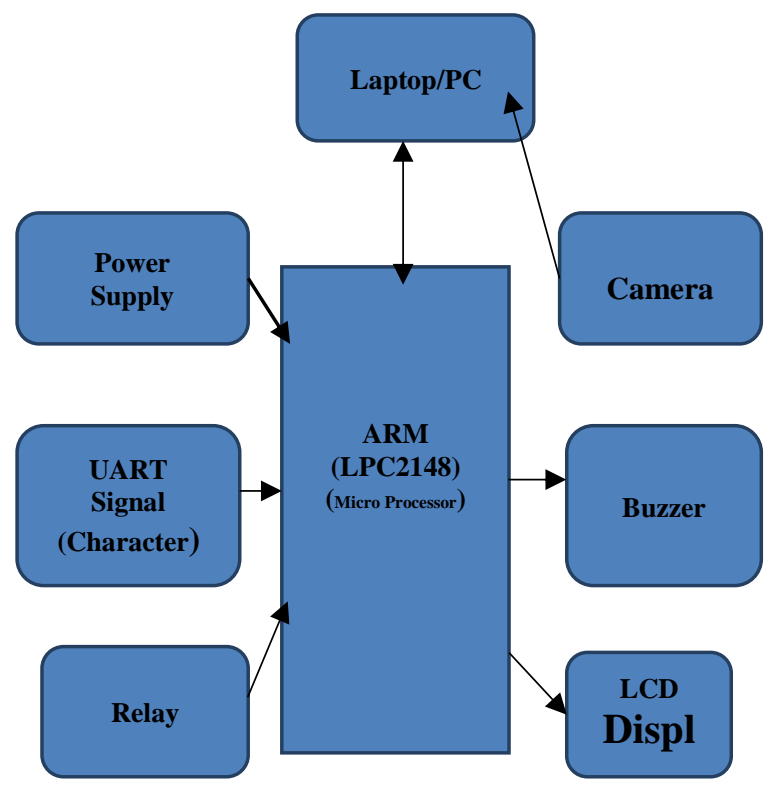

Figure 1. Block diagram

\subsection{Iris Recognition Concept}

This research is basically on an electronic device which can be used in emergency to prevent the accident. The main idea behind this is to develop a system which can detect fatigue of the driver and issue a timely warning so as to avoid the accident. According to the survey, large number of road accidents occur due to the driver drowsiness, hence this system will be helpful in preventing the accidents, and consequently reduce personal sufferings. This system will monitor the driver eyes using a camera and with the help of an algorithm developed, it can detect symptoms of driver fatigue early enough to avoid an accident. So this will be helpful in detecting driver fatigue in advance and will give warning output in the form of sound.

Here the Iris recognition is used to detect the driver drowsiness. Driver drowsiness is one of the main causes for the road accidents. The driver's drowsiness detection can reduce accidents. In this paper, an algorithm is proposed to determine open or closed state of the driver's eye, based on the iris area of the eye in open or close state. In the proposed method, the state of the eye is being monitored throughout. The result shows that the proposed algorithm has sufficient speed, accuracy, and less complexity, so it can be used in the real time. 


\section{RESEARCH METHOD}

\subsection{Hardware}

A hardware model has been developed to implement the above scheme. A web camera with IR illuminators has been employed for focusing the face region of the driver to acquire the images of the face. The acquired image signal is fed to Data Acquisition Card and subsequently to ARM Processor.

The ARM Processor analyses the images and detects the iris characteristics. If the eye is closed continuously for four seconds, it may be assumed that the driver is under drowsiness and the alarm is activated by the ARM Processor. ARM Processor LPC2148 is employed here in association with voltage regulator IC 7805 and driver IC L2930 for the buzzer. To find the position of iris, the eye region must be separated from the rest of the image using rectangle function, which is a process of segmentation. This will cause the images background to be non-effective. This will results in decreasing the computational complexity. Finally in the proposed method, circles selected as the driver's eye condition using Hough transformation function. The iris region is well detected. If eye is open, then the next frame is simulated and if the eye is closed for continuously for four seconds, then a signal is passed to the ARM Processor for raising the alarm. This is how the system can awake the driver in long drive or in fatigue condition.

\section{FLOW CHART}

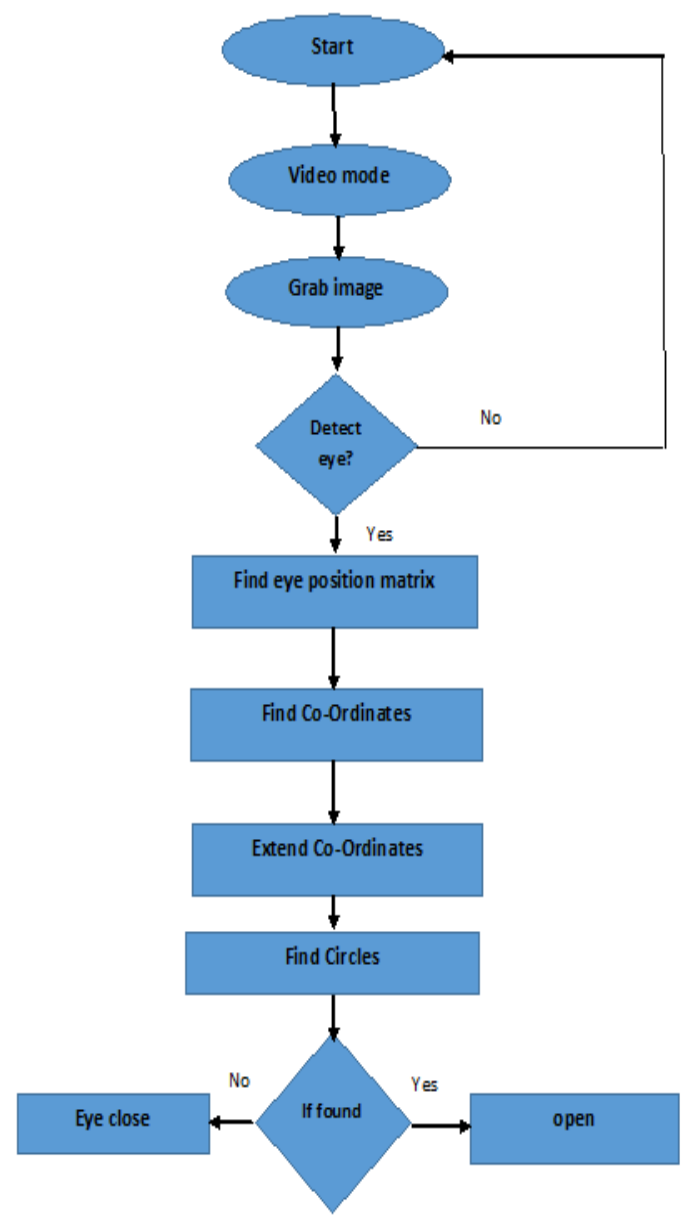

Figure 2. Flow chart for the Eye detection

\subsection{Face Detection}

Numerous approaches have been proposed for detecting face. Face detection based on mat lab, Viola-Jone algorithm and skin colour detection method are most commonly used and famous approaches for face detection neural network based method is most accurate. It consists of learning and performing stages. In the former stage the system is learned from a huge set of training images to identify skin and non-skin 
coloured pixels. This knowledge is used when an unknown image is given. Accuracy is appreciable for this method but it is time consuming. Viola-Jones algorithm overcomes this difficulty.

\subsection{Eye Detection}

The next step is eye detection from the detected face region. Here a new method is suggested for eye detection. It is based on the assumption that, the left eye is at the left upper corner of face and right eye is at the right upper corner of face region. After detecting the face, crop the face as a rectangular region. The left and right eyes are located in a rectangular region having width $1 / 3$ width of face region. By knowing the coordinates of face region, eye region coordinates can be calculated by the following equations.

Equation for left eye:

$$
\text { eye } 1,:=\text { out } 1,1+\operatorname{siz} * 0.58, \text { out } 1,2+\operatorname{siz} * 0.28, \text { siz* } 0.22, \operatorname{siz} * 0.22 \text { (2) }
$$

Equation for Right eye:

$$
\text { eye } 2,:=\text { out } 1,1+\operatorname{siz} * 0.2 \text {, out } 1,2+\operatorname{siz} * 0.28, \operatorname{siz} * 0.22, \operatorname{siz} * 0.22
$$

where, eye[] is a $2 \times 4$ matrix, in which first row will store left eye corner coordinates and second row contains right eye coordinates, out[] is $1 \times 4$ matrix which contains face region corner coordinates and "siz" is the width of face region extracted.

\subsection{IRIS Recognition}

Iris detection is the major phase in drowsiness detection. So this phase must have appreciable accuracy. Here circular Hough transform is choosen for this purpose. The voting procedure is carried out in a parameter space, from which object candidates are obtained as a local maxima in an accumulator space that is explicitly constructed by the algorithm for computing the Hough transform.

In this system, cropped eye image and maximum and minimum radius of iris is given as input for taking circular Hough transform. For an adult human, average radius of iris is between $5 \mathrm{~mm}$ and $7 \mathrm{~mm}$. So, 10 and 25 pixel points as maximum and minimum radius of iris is used here. As an initial step, edge detection is performed. Canny edge detection is used because it detects strong and weak edges and includes the weak edges in the output only if they are connected to strong edges.

Hough transform can be used to find any geometrical figure in an image. More complex shapes are described using parameters. A circle might seem easy to represent using three parameters. The equation of a circle is $r^{2}=(x-a)^{2}+(y-b)^{2}$ and it has three parameters $r$, $a$ and $b$ where, $r$ is the radius of circle, $a$ and $b$ are the centre in $\mathrm{X}$ and $\mathrm{Y}$ direction. The parameter space of a circle belongs to $\mathrm{R}^{3}$. Now we need to convert the edge detected binary image to the parameter space. This is easy since the pixel coordinate can be directly translated to $\mathrm{a}$ and $\mathrm{b}$ in the parameter space. If the radius is known, a constant radius may be used to simplify the computation. Otherwise a finite list of radii must be evaluated. Radius of iris is different from person to person, but it is in a specified range.

This paper suggests the second method. For each radius find out the coordinate positions, which have non zero value in both edge image and circle with that radius. If such a pixel position is detected, increment the value corresponding to that position in accumulator space. After complete voting, find out the maximal points and number of non-zero valued pixels in the circumference of the circle with the maximal point as centre. The circle having larger number of such points in its circumference is the iris. Figure 3 shows the detected iris.

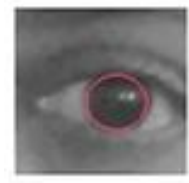

Figure 3. Detected iris

\subsection{Drowsiness detection}

Drowsiness can be detected based on the visibility ratio of iris. Iris is not visible completely in two situations, ie, during blinking of eye and in drowsy condition. Differentiate these two is the next task. Here, if the iris visible ratio is less than $60 \%$, then that frame will be considered for further analysis. ie, to 
detect it is blink or drowsiness. For that, analyse the previous frame. If it also in the same condition, consider the situation as drowsy otherwise it is just a blink. Figure 4 shows the Drowsiness detection.

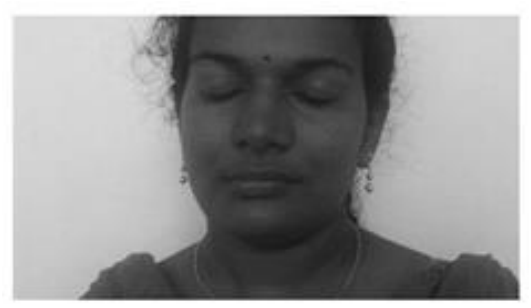

Figure 4. Drowsiness detection

\section{RESULTS AND PERFORMANCE EVALUATION}

5.1. Initial Condition

Figure 5 shows the initial condition picture captured by web camera when eye is open, it shows no problem on matlab command.

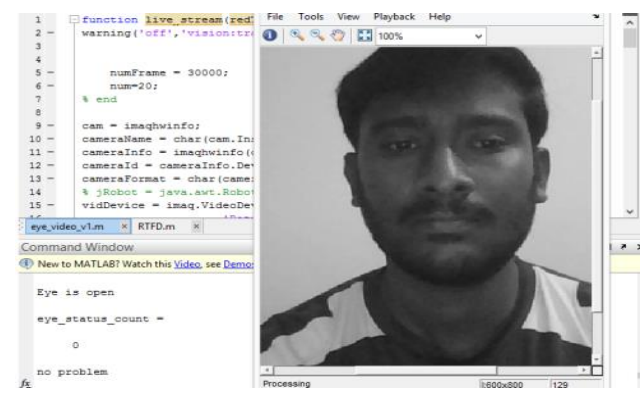

Figure 5. Initial Condition

\subsection{Second condition}

Figure 6 shows the condition when eyes closed for $1^{\text {st }}$ count (one Second) When eye is closed, it starts to count for few seconds. For the first count, the status of eyes is" eye closed" but the result shows no problem in first condition, we cannot predict it as danger so it will take more picture or frames.

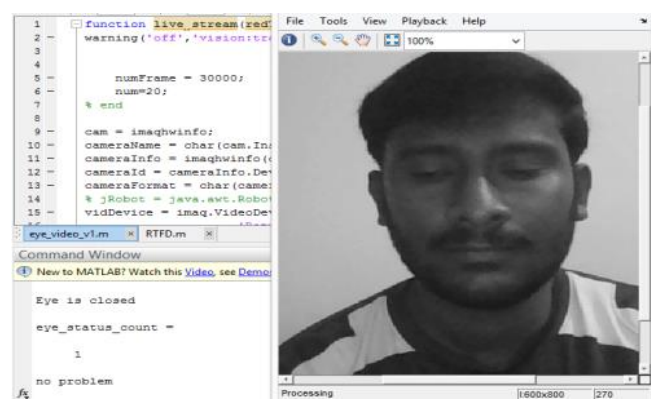

Figure 6. When eyes closed for $1^{\text {st }}$ count

\subsection{Third Condition}

In the third condition, when eyes are closed for $4^{\text {th }}$ count (Eye is closed continuously for four Seconds), as shown in Figure 7, it shows as danger and will send a signal to the Processor to give warning message. 


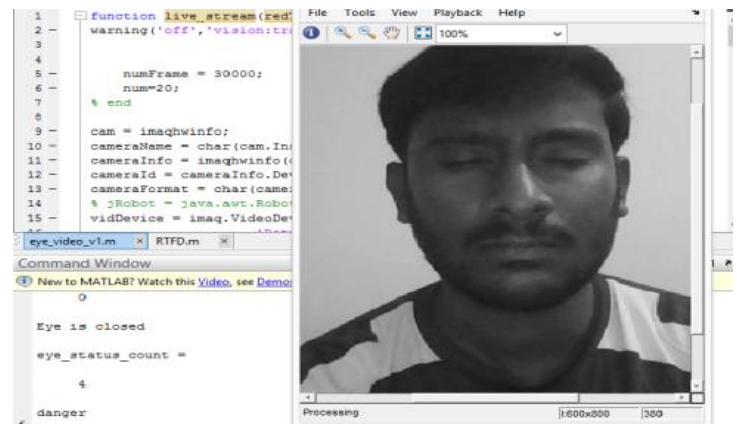

Figure 7. When eyes closed for $4^{\text {th }}$ count

\section{CONCLUSION}

The analysis and design of real time driver fatigue detection system is carried out. The proposed system provides the detection of driver fatigue. It is used to avoid the road accidents caused by drowsy driving and it can also help drivers to be awake while driving, by giving a warning when the driver is sleepy. And also this system can be used for security purpose of a driver.

During the monitoring, the system is able to decide if the eyes are open or closed. When the eyes have been closed continuously for four seconds, a warning signal is issued. Image processing achieves highly accurate and reliable detection of drowsiness. This is achieved by interfacing a web camera to a PC and recording test videos and frame database under different lighting conditions. The calculation speed, accuracy and robustness will be influenced by using algorithm and hardware part.

\section{ACKNOWLEDGEMENTS}

I would like to take this opportunity to express my sincere gratitude to Mr.Aswathnarayana $\mathrm{P}$, Mr.Sagar N, Ms.Bharathi C and Ms.Shilpa Rani, the students of BMSIT, Bangalore for helping me in carrying out this project. Also, I would like to thank Jain University for providing me the overall support in my academic and research activities.

\section{REFERENCES}

[1] V. Phanikumar S., et al., "Face recognition with modular two dimensional PCA under Uncontrolled illumination varitions," International journal of Electrical and Computer Engineering, vol/issue: 6(4), pp. 1610-1616, 2016.

[2] V. Kumar, et al., "Accurate iris localization using edge map generation and adaptive circular Hough transform for less constrained iris images," International journal of Electrical and Computer Engineering (IJECE), vol/issue: 6(4), pp. 1637-1646, 2016.

[3] Secretary, Government of India, Ministry of Road Transport and High Ways, "Road Accidents in India-2015," Transport Research Wing Report, 2015.

[4] N. Kuamr and N. C. Barwar, "Analysis of Real Time Driver Fatigue Detection Based on Eye and Yawning," International Journal of Computer Science and Information Technologies, vol/issue: 5(6), pp. 7821-7826, 2014.

[5] A. Sengupta, et al., "Analysis of Fatigue using EEG State-Space Analysis," Annual IEEE India Conference (INDICON), 2014.

[6] W. He, "Research on Heart Rate Variability to Driver Fatigue Detection of Dangerous Chemicals Vehicles Based on simulation analysis," 7th International Conference on Intelligent Computation Technology and Automation, Changsha, Hunan, China, 2014.

[7] M. Mischi, et al., "Analysis of muscle fatigue induced by isometric vibration exercise at varying frequencies," 34th Annual International Conference of the IEEE EMBS San Diego, California USA, 2012.

[8] B. G. Patil, et al., "Iris feature extraction and classification using FPGA," International journal of Electrical and Computer Engineering (IJECE), vol/issue: 2(2), pp. 214-222, 2012

[9] M. M. Bundele and R. Banerjee, "ROC Analysis of a Fatigue Classifier for Vehicular Drivers," Intelligent Systems (IS), 5th IEEE International Conference, 2010.

[10] K. Sekar, et al., "Dynamically configurable bus topologies for high-performance on-chip communication," IEEE Trans. Very Large Scale Integr. (VLSI) Syst., vol/issue: 16(10), pp. 1413-1426, 2008.

[11] J. Shen and P. Hsiung, "Dynamic Reconfigurable Network-on-Chip Design: Innovations for Computational Processing and Communication," J. Shen and P. Hsiung, Eds. Hershey, PA, USA: IGI Global, 2010.

[12] G. M. Chiu, "The odd-even turn model for adaptive routing," IEEE Trans. Parallel Distrib. Syst., vol/issue: 11(7), pp. 729-738, 2000.

[13] S. Jovanovic, et al., "A new deadlock-free fault-tolerant routing algorithm for NoC interconnections," in Proc. Int. Conf. Field Program. Logic Appl., pp. 326-331, 2009. 
[14] W. Dally and C. Seitz, "Deadlock-free message routing in multiprocessor interconnection networks," IEEE Trans. Comput., vol/issue: C-36(5), pp. 547-553, 1987.

[15] C. Bobda, et al., "DyNoC: A dynamic infrastructure for communication in dynamically reconfigurable devices," in Proc. Int. Conf. Field Program. Logic Appl., pp. 153-158, 2005.

[16] T. Pionteck, et al., "Applying partial reconfiguration to networks-on-chip," in Proc. Field Program. Logic Appl. Int. Conf., pp. 1-6, 2006.

[17] S. Jovanovic, et al., "A new high-performance scalable dynamic interconnection for fpga-based reconfigurable systems,” in Proc. Int. Conf. Appl.-Specific Syst., Archit. Process., pp. 61-66, 2008.

[18] S. Jovanovic, et al., "CuNoC: A dynamic scalable communication structure for dynamically reconfigurable FPGAs," Microprocess. Microsyst, vol/issue: 33(1), pp. 24-36, 2009.

\section{BIOGRAPHIES OF AUTHORS}
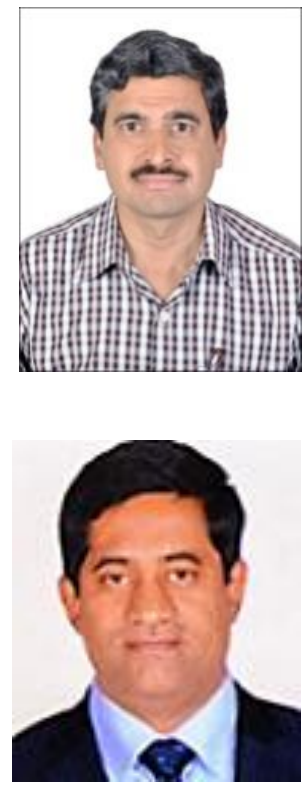

Gopalakrishna K has awarded the B.E degree in Electronics from Bangalore University, M.Tech degree in VLSI Design and Embedded Systems from VTU, M. Phil degree in Computer Science from M.S.University, Tirunelveli, Tamil Nadu and pursuing PhD. from Jain University. He has 23 years of teaching experience. He is currently working as Associate Professor, Dept. of Electronics \& Communication Engineering, School of Engineering \& Technology, Jain University. His field of interests includes Advance microprocessors, Microcontrollers, Embedded Systems, VLSI Technology and VLSI Designing.

Dr. Hariprasad obtained B.E degree in the year 1991 and M.E in the year 2000.He has completed Ph.D. degree in the year 2011 and two additional highest degrees, Doctor of science degrees in the year 2013 and 2014 for the post-doctoral research work on communication and embedded systems. He has published 60 papers in International and National Journals/conferences and a text book on "Advanced microprocessors". Dr. Hariprasad has 22 years of teaching experience and 09 years of research experience. He held key posts, like Director for Cognitive science, RVCE, Vice-principal, BMSIT, Pro-vice-Chancellor-Dayananda Sagar University, Academic mentor for DSCE and DSATM. Dr. Hariprasad established industry supported labs and served as visiting professor to various reputed colleges in Bangalore. Currently he is working as Director, School of Engineering Technology, Jain University. 